\title{
Electromagnetic Field Intensity Imaging by Thermofluorescence in the Visible Range
}

\author{
Stéphane Faure, ${ }^{1}$ Jean-François Bobo, ${ }^{2}$ Daniel Prost, ${ }^{3}$ François Issac, ${ }^{3}$ and Julian Carrey ${ }^{1, *}$ \\ ${ }^{1}$ Laboratoire de Physique et Chimie des Nano Objets, LPCNO, UMR 5215 INSA-UPS-CNRS, Université de \\ Toulouse, 31077 Toulouse, France \\ ${ }^{2}$ Centre d'Élaboration de Matériaux et d'Etudes Structurales (UPR 8011), CEMES CNRS, 29 rue Jeanne Mavig, \\ 31055 Toulouse, France \\ ${ }^{3}$ Office National Etudes Recherches Aérospatiale, ONERA, DEMR, 2 avenue Edouard Belin, \\ 31000 Toulouse, France
}

(Received 14 July 2018; revised manuscript received 22 January 2019; published 30 May 2019)

\begin{abstract}
Methods to measure the electromagnetic field intensity on a large scale should be improved to meet the growing demand in civil and military applications. The recent development and an application of a microwave electromagnetic field imaging method using a thermofluorescent device and detection in the visible-light range are described. The sensor is a flexible film sensitive to either an electric field (slightly conductive film) or a magnetic field (insulating film with ferromagnetic particles) coated with a polymer doped with fluorescent molecules displaying temperature-dependent emission. We use a lock-in thermography protocol consisting of modulation of the field at low frequency, which allows us to suppress the noise due to heat-transfer phenomena and the part of the signal not directly related to field absorption. Two applications of the method are presented: (i) imaging the microwave magnetic field emitted by a resonator antenna and (ii) the local probe measurement of the electric field inside a multibeam antenna. The process to develop the sensitive film, its detailed characterization, and the thermofluorescence-image demodulation postprocessing are presented in detail. This approach is much cheaper than one based on infrared thermometry and could permit democratization of microwave- and radio-frequency electromagnetic field imaging.
\end{abstract}

DOI: 10.1103/PhysRevApplied.11.054084

\section{INTRODUCTION}

The omnipresence of radio-frequency (rf) electromagnetic devices in our environment leads to an increasing demand to probe, measure, and visualize electromagnetic (em) fields. Applications range from mainstream markets (telecommunications, printed circuit boards, global induction hobs) to specific technologies (radars, aeronautics, healthcare, etc.). The two-dimensional (2D) and threedimensional numerical calculations of EM fields with finite-element methods combined with meshing algorithms offered until recently a cheaper and more-convenient way than the fabrication of probe tools. However, simulations can be time-consuming or very costly in terms of memory, especially with complex systems. Moreover, the results of the simulations can be inaccurate due to the high entanglement of the multiphysics problem or poor knowledge of the material properties used as entry parameters in the algorithm.

\footnotetext{
*julian.carrey@insa-toulouse.fr
}

A method permitting the real-time quantitative cartography of EM fields would be interesting for diagnosis and characterization (e.g., fast visualization of antenna field patterns) [1]. However, alternating electric field and magnetic field direct measurements are usually spatially localized [2,3], so space scanning is required for mapping $[4,5]$. On the other hand, IR thermography imaging is a quite-developed method for nondestructive testing and electromagnetic compatibility studies [6-8]. Only recently has imaging of EM fields by combination of IR thermometry and $\mathrm{rf}$ and/or microwave absorbents demonstrated at ONERA [1,9-11]: the so-called electromagnetic IR (EMIR) method uses a conductive film sensitive to EM microwaves; the Ohmic losses due to induced currents create a measurable heating that is detected by an IR camera. It gives an image of the electric field pattern and amplitude. The use of microstructured films with large ferromagnetic losses extends this method to the measurement of the magnetic component of the field [12]. To improve the quality of the measurements, lock-in thermography can be used to suppress all phenomena not directly related to the excitation source. This method has been applied for many years in nondestructive testing of electronic devices as 
a safe and nonintrusive method and is well documented $[13,14]$.

IR cameras are quite expensive compared with visible CMOS detectors, typically several thousand euros for a CMOS sensor and several tens of thousands of euros for an IR sensor A comparison of the prices for both technologies is given in Table SI1 in the Supplemental Material [15]. Moreover, IR thermography has some disadvantages: saturation of the detector at high temperatures, difficulties to estimate precisely the emissivity of the surface materials, and a spatial resolution for the temperature detection which is Rayleigh limited. The development of films sensitive to both the electric component and the magnetic component of the field, coupled with a cheap and easy-to-transport detector, would be very attractive.

In the present case, our objective is to replace IR thermography by thermofluorescence spectroscopy (TFS) so as to be able to perform measurements with a camera much cheaper than IR cameras and to increase the spatial resolution of the field images obtained. Fluorescence-based thermometry, applied in a wide range of research and industrial applications, uses the fluorescence properties of molecules (fluorophores) that are usually addressed to a target receiver (deposited on an absorbent film in our case) to derive the temperature [16,17]. During a TFS experiment, part of the incident EM field absorbed by the film is transformed into heat by the absorbent. This part should be sufficiently weak (about $15 \%$ of the EM power) so as not to disturb the field. The fluorophores under visible or near-UV excitation emit light in the visible spectrum at a longer wavelength with a quantum efficiency that depends on temperature. If one modulates the field amplitude, the temperature generated in the sample fluctuates at the modulation frequency, resulting in the modulation of the fluorescence signal. Signal postprocessing enables one to derive the amplitude and phase angle of the thermal waves. Knowing precisely the absorption properties of the absorbent film permits one to derive quantitatively the field amplitude.

We recently designed such sensitive films and successfully demonstrated the possibility to image in the visible range the microwave magnetic field emitted in the near field of a zeroth-order resonator (ZOR) antenna in the visible range [18].

We recently patented this new method [17] and named it EMVI (for "electromagnetic visible imaging"). In the present work, the fabrication and characterization of the thermofluorescent film, the theory of heat transfer, and different aspects of the signal modulation and demodulation are presented. Then two kinds of application of the method are shown: field thermography and field local probing. Comparison between magnetic field imagery of a microwave-frequency antenna obtained by IR thermography and the TFS method as well as local measurements of the electric field inside a multibeam microwave antenna are presented. Finally, the merits and limits of the method are discussed.

\section{SENSOR DEVELOPMENT AND CHARACTERIZATION}

\section{A. Choice of the fluorescent material}

Two kinds of absorbent film are used in this study: (i) Kapton ${ }^{\circledR}$ resistive thin films with low electrical conductivity (the induced electric currents generate Ohmic losses resulting in film heating); (ii) wide-frequency magnetic absorbent films commercialized by the Japanese company NEC Tokin. These films are mainly designed for telecommunication devices as suppressors for the high-frequency noise generated by electronic devices. They have a low conductivity $(\sigma)$ and high permeability $(\mu)$. The flexible sheet is a polymer inside which blended micron-sized magnetic particles are dispersed. Interaction with the magnetic component of the field generates magnetic losses (due to the imaginary part of the permeability) and thus heat. Some useful properties of both films are summarized in Table SI2 in the Supplemental Material [15].

An acetate cellulose thin layer doped with a fluorophore is then deposited on the absorbent film by spin coating. Preliminary tests lead us to choose rhodamine B as the fluorophore, for the following reasons: high quantum efficiency $[19,20]$, good spectral separation of absorption and emission spectra, good thermal sensitivity (approximately $1 \% \mathrm{~K}^{-1}$ ), and cheapness (which permits intensive testing). The acetate cellulose adheres well to the Tokin film surface, which has substantial roughness (several tens of microns). However, the polymer adheres poorly to the Kapton film surface. The compatible combination of a fluorophore, a solvent, and a polymer with good adherence to the target surface is difficult to predict a priori. The choice is made on a trial-and-error basis. Optical images of the samples show a homogeneous surface [see Figs. SI1(a) and SI1(b) in the Supplemental Material [15]. The thickness of the film is estimated with a scanning electron microscope. The Tokin film thickness is approximately $280 \mu \mathrm{m}$, in good agreement with the $300 \mu \mathrm{m}$ expected from the data sheet (see Fig. 1). The fluorescent-layer thickness ranges between 40 and $76 \mu \mathrm{m}$. When the fluorescent film is deposited on Kapton, the typical thickness is less than $100 \mu \mathrm{m}$.

\section{B. Fluorophore calibration}

All samples are calibrated with the same protocol (see "Experimental Section" in the Supplemental Material [15]). We present here the case of a Tokin film coated with rhodamine B embedded in acetate cellulose polymer. Fluorophore calibration is done by our heating a sample with a hot plate and measuring the fluorescence in the steady regime with an optical fiber probe [see Fig. 2(a)]. The fluorescence peak area decreases linearly 


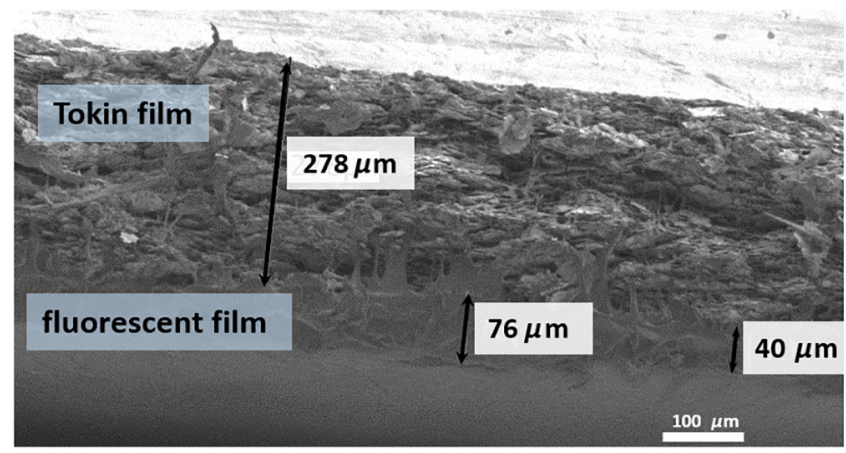

FIG. 1. Cross-section scanning-electron-microscope micrographs of Tokin capped by a thin fluorescent film.

with temperature in the range from 290 to $350 \mathrm{~K}$ (see Fig. SI2 in the Supplemental Material [15]). A linear trend is observed until $550 \mathrm{~K}$ (not shown). A calibration function is deduced by a linear fit of the function [see Fig. 2(b)]. The relative sensitivity defined by $S_{r}=$ $(d \mathscr{A} / d T) / \mathscr{A}$ is measured to be $1.8 \% \mathrm{~K}^{-1}$ where $\mathscr{A}$ is the fluorescence peak area. A comparison between the temperature deduced from the fluorescence and the temperature measured by the pyrometer over five temperature cycles between 295 and $315 \mathrm{~K}$ shows very good agreement in intensity and dynamics [see Fig. 2(c)]. We observe increasing disagreement between the pyrometer-measured temperature and the fluorescence-deduced temperature as a function of time. It is characteristic of the photobleaching effect due to long exposure of the fluorophore to light. These experiments validate rhodamine $\mathrm{B}$ as a suitable thermometer.

\section{HEAT TRANSFER THEORY UNDER MODULATED EXCITATION}

\section{A. Electric and magnetic losses}

To measure the electric field $E$, we use a lowconductivity film (Kapton film with a thickness of about $50-100 \mu \mathrm{m}$ ) weakly coupled to the electric field but with enough induced currents to get measurable heating. Under specular microwave excitation, the Kapton film absorption rate $A$ is related to the surface impedance $Z_{S}$ (or surface resistance) [21]:

$$
A=\frac{4 Z_{S} Z_{0}}{\left(Z_{0}+2 Z_{S}\right)^{2}}
$$

where $Z_{0}$ is the vacuum impedance $\left(Z_{0}=377 \Omega\right)$ and $Z_{S}$ is defined as

$$
Z_{S}=\frac{1}{\sigma e_{1}}
$$

where $\sigma$ is the film electric conductivity and $e_{1}$ is the film thickness. The film thickness $e_{1}=50 \mu \mathrm{m}$ is chosen in such a way that it is much lower than the skin depth (see below). In the case of a film with absorption $A=10 \%, Z_{S}$ is $2-3 \mathrm{k} \Omega$.

The density of absorbed power $P_{\mathrm{abs}}\left(\mathrm{W} \mathrm{m}^{-3}\right)$, in the case of microwave propagation at frequency $f$ is given by

$$
P_{\mathrm{abs}}=\frac{2 Z_{S}}{e_{1}\left(Z_{0}+2 Z_{S}\right)^{2}} E^{2} .
$$

(a)
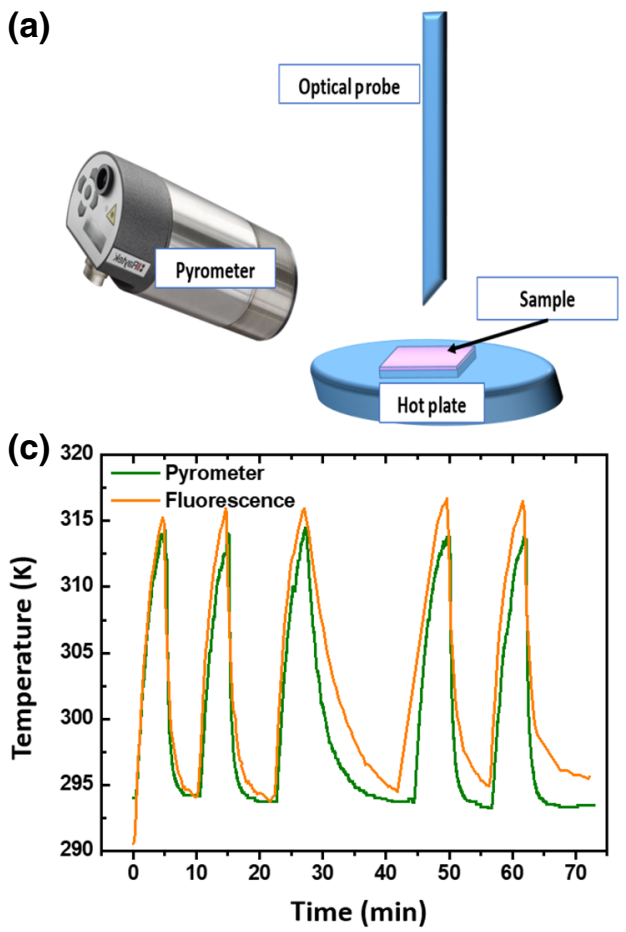

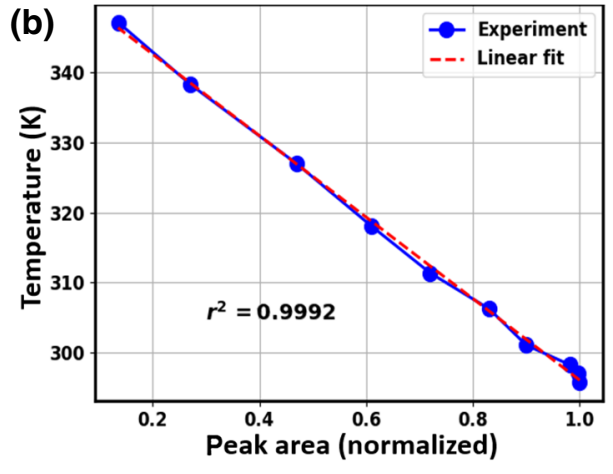

FIG. 2. (a) Hot-plate-calibration setup. (b) Evolution of the total peak area as a function of temperature. The area is normalized by its value at $295.8 \mathrm{~K}$. The solid red line is a linear fit of the experimental data. (c) Comparison of the temperature measured by TFS and by the pyrometer. 
To measure a magnetic field $H$, we use a high-permeability film (Tokin film). The power absorbed by the magnetic particles (hysteresis cycle area) is proportional to the imaginary part of the permeability $\mu^{\prime \prime}$, the field frequency $f$, and the square of the magnetic field $H$ [12]:

$$
P_{\mathrm{abs}}=\pi f \mu^{\prime \prime} H^{2} .
$$

In the case of a thermofluorescent thin film made of two layers [i.e. for a thickness $e=e_{1}+e_{2}$ ( $e_{2}$ the fluorescentlayer thickness) small compared with the wavelength and penetration depth $(e \ll \lambda, \delta)]$, the film heating at thermal equilibrium $\Delta T_{\text {avg }}$ is related to the density of absorbed power $P_{\text {abs }}\left(\mathrm{W} \mathrm{m}^{-3}\right)$ by

$$
\Delta T_{\mathrm{avg}}=\frac{P_{\mathrm{abs}} e}{2 h},
$$

where $h$ is the heat-transfer coefficient $\left(\mathrm{W} \mathrm{m}^{-2} \mathrm{~K}^{-1}\right)$ between the film and the environment.

Equation (5) is valid for both electric field absorption and magnetic field absorption.

In the case of electric field measurement, the temperature varies as the square of the amplitude of the electric field $E$, so

$$
|E|=k_{E} \sqrt{\Delta T_{\mathrm{avg}}},
$$

where $k_{E}=\left[\left(2 h / e_{1}\right)\left(Z_{0}+2 Z_{S}\right)^{2} / 2 e_{1} Z_{S}\right]^{1 / 2}$, a constant of proportionality. In the case of magnetic field measurement, the temperature varies as the square of the magnetic field $H$ :

$$
|H|=k_{H} \sqrt{\Delta T_{\text {avg }}},
$$

where $k_{H}=\left(2 \pi e_{1} f \mu^{\prime \prime} / c\right)^{1 / 2}$.

\section{B. Heat-transfer theory}

After the sample is heated by an EM source, the energy absorbed by the sample is converted into heat and the heat spreads into the sample. This process is governed by the thermal-diffusion equation. Along the $z$ axis perpendicular to the film plane (see Fig. 3), the thermal-diffusion length $D$ when the heating source is modulated at frequency $\omega$ is given by [22]

$$
D=\sqrt{\frac{2 \alpha}{\omega}}=\sqrt{\frac{\alpha}{\pi f}},
$$

where $\alpha=\kappa / \rho C_{p}\left(\mathrm{~m}^{2} \mathrm{~s}^{-1}\right)$ is the thermal diffusivity of the film, $\rho\left(\mathrm{kg} \mathrm{m}^{-3}\right)$ is the mass density, $C_{p}\left(\mathrm{~J} \mathrm{~kg}^{-1} \mathrm{~K}^{-1}\right)$ is the specific heat, and $\kappa\left(\mathrm{W} \mathrm{m}^{-1} \mathrm{~K}^{-1}\right)$ is the thermal conductivity. The thermal-diffusion length is about $50-500 \mu \mathrm{m}$ in Kapton and Tokin sheets for modulation frequency ranging between 0.1 and $10 \mathrm{~Hz}$. As a consequence, we have the

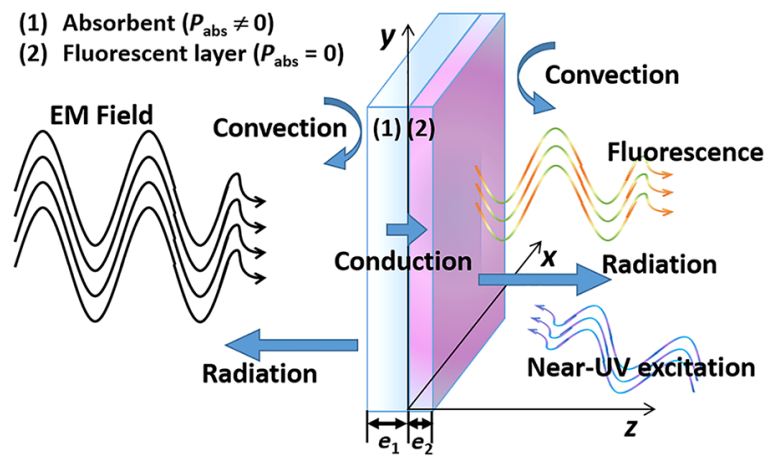

FIG. 3. EM-field-imagery mechanisms. A fraction of the field is absorbed by the sensitive film (1) and transformed into heat. Heat diffuses through the film and part is transmitted to the fluorescent layer (2). Another part of the heat is evacuated by convection and radiation. The fluorescence properties are directly related to temperature.

case of a 2D film with thickness $e<D$, so the heat equation can be simplified as

$$
\rho C_{p} \frac{\partial T}{\partial t}=P_{\mathrm{abs}}(t)-\frac{2 h}{e}\left(T-T_{\mathrm{env}}\right)
$$

where $T$ is the temperature (K), $P_{\text {abs }}$ is the incident power absorbed by the film (W), $h$ is the heat-transfer coefficient $\left(\mathrm{W} \mathrm{m}^{-2} \mathrm{~K}^{-1}\right)$ between the film and the environment, $e$ is the film total thickness (m), and $T_{\text {env }}$ is the temperature of the environment $(\mathrm{K})$. The factor 2 accounts for the two sides of the film. In the case of different surfaces, the factor $2 h$ should be replaced by $h_{1}+h_{2}$. Radiation losses can be ignored for weak heating. The right term in Eq. (9) corresponds to Newton's law of cooling related to the temperature gradient occurring between the environment and the film surfaces, creating losses by convection.

In the case of a film coated with fluorophores embedded in a polymer, we assume a thermally ideal interface between the two materials so that the temperature and heat flow are continuous. The boundary conditions for heat diffusion through such an interface are given by

$$
T_{1}=T_{2},
$$

where the subscripts 1 and 2 refer to the two different regions in thermal contact. In the case of a timeindependent heat source ( $P_{\mathrm{abs}}$ is constant), the temperature evolution is given by

$$
T(t)=T_{\mathrm{env}}+\Delta T_{\mathrm{avg}}\left(1-e^{-(t / \tau)}\right) .
$$

The thermal steady state is reached after a few seconds, corresponding to the time constant of the film $\tau=$ $\rho C_{p} e / 2 h$. 


\section{Demodulation process}

A good way to maximize the signal-to-noise ratio in a thermography experiment is to modulate the excitation $[7,8]$. The detected signal is mixed with noise (typically background noise, disturbing reflections, or heat diffusion at or on the sample surface) that fluctuates slowly with time. If the heating source is modulated by a square wave of frequency $f_{\text {mod }}$, the absorbed power $P(t)$ for one period of the modulation can be written as

$$
P(t)= \begin{cases}P_{\mathrm{abs}}, & |t|<\frac{w}{f_{\text {mod }}}, \\ 0, & \frac{w}{f_{\text {mod }}}<|t|<\frac{1}{f_{\text {mod }}} .\end{cases}
$$

The factor $w \in[0,1]$ depends on the duty cycle. For example, $w=0.5$ for a $50 \%$ duty cycle. So, in the general case, the time evolution of the temperature is a succession of exponential growth and decay functions. However, if the modulation period is small compared with the time constant of the film $\tau$, the exponential decay and growth reduce to linear slopes, so the time evolution of the temperature can be written as

$$
T(t)=T_{\mathrm{env}}+\Delta T_{\mathrm{avg} \bmod }\left(1-e^{-(t / \tau)}\right)+\frac{\Delta T_{\mathrm{mod}}}{2} \Lambda(w, \phi),
$$

where $\Delta T_{\text {mod }}$ and $\phi$ are the peak-to-peak amplitude and phase angle of the triangle function $\Lambda(w)$ of duty cycle $w$. Figure 4 shows an experimental curve illustrating heating under a modulated excitation. During modulation, the equilibrium temperature $\Delta T_{\text {avg mod }}$, around which the temperature oscillates, is reduced compared with the case where the source excites the film continuously. We have

$$
\Delta T_{\text {avg mod }}=\Delta T_{\text {avg }} w,
$$

where $\Delta T_{\text {avg }}$ is the temperature that is reached in the absence of modulation (see Fig. 4). In the case shown in Fig. 4, the steady state is reached after a few tens of seconds.

Moreover, it can be shown easily that (see Supplemental Material [15])

$$
\Delta T_{\bmod }=P_{\mathrm{abs}} \frac{w(1-w)}{\rho C_{p} f_{\bmod }} .
$$

In the case of a 2D thin film stimulated by an EM field modulated with a sine waveform instead of a rectangular one,

$$
P_{\mathrm{abs}}(t)=\frac{1}{2} P_{\mathrm{abs}}(1+\sin \omega t) .
$$

The theoretical solution given by Eq. (9) becomes

$$
\begin{aligned}
T(t)= & T_{\mathrm{env}}+\frac{1}{2} \Delta T_{\mathrm{avg}}\left(1-e^{-(t / \tau)}+\frac{2 h}{\sqrt{4 h^{2}+\left(\rho C_{p} e \omega\right)^{2}}}\right. \\
& \left.\times\left[\sin (\omega t-\varphi)+\sin \varphi \times e^{-(t / \tau)}\right]\right),
\end{aligned}
$$

with $\tan \varphi=\rho C_{p} e \omega / 2 h$ the phase shift due to the thermal inertia of the film.

It follows that in the steady state and for a thin film for which the phase shift is ignored, the heating amplitude at frequency $\omega$ is given by [23]

$$
\Delta T_{\mathrm{mod}}=\frac{P_{\mathrm{abs}} e}{\sqrt{4 h^{2}+\left(\rho C_{p} e \omega\right)^{2}}} .
$$

Equation (15) is consistent with Eq. (18) in the limit of a long modulation period and weak heating (see Supplemental Material [15]). It is also a good approximation for $\Delta T_{\text {mod }}$ in the case of a rectangular modulation.

Equations (14), (15), and (18) establish a link between film heating in the case of continuous and modulated excitation and the EM-field amplitude via fluorescence variation. Depending on the film absorption rate and characteristic parameter $\tau$, it could be more convenient to modulate the field by varying the duty cycle $w$ than the

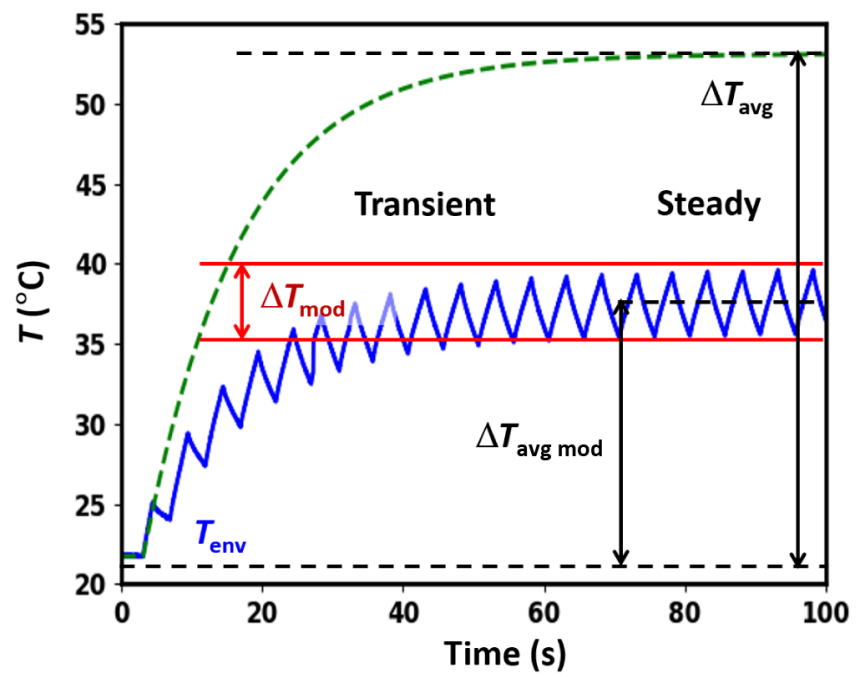

FIG. 4. Tokin-film heating under constant (dotted green line) and $0.2-\mathrm{Hz}$ modulated (blue line) magnetic excitation measured by IR thermography. The average heating $\Delta T_{\text {avg }}$ is equal to the difference between the average temperature at thermal equilibrium and the initial temperature. The heating under modulation $\Delta T_{\text {mod }}$ is equal to the amplitude of the temperature modulation in the steady state. The average heating under modulation $\Delta T_{\text {avg mod }}$ represents the average heating reached in the steady state. 
modulation frequency $f_{\text {mod }}$ so as to avoid burning the film or saturating the detector.

We now illustrate the way $\Delta T_{\text {mod }}$ is extracted from experimental data where the sample is submitted to a modulated excitation. First, two problems are mentioned: (i) Some experimental artefacts (e.g., electronic jitter, clock drift, or frame skipping) lead to a slight difference between the experimental modulation frequency of the signal generator and the modulation frequency of the measured signals. This prevents good convergence of the FFT algorithm used to demodulate the signal (see below) [24]. (ii) If the sample is excited with a rectangular excitation, the demodulated amplitude given directly by the IR camera cannot be trusted since the camera demodulation is done by an ana$\log$ process that gives the amplitude of the first harmonic of the FFT; this is not enough since the sample responds with a triangle function [see Eq. (13)]. This mode should be used when the sample is excited with a sinusoidal excitation only or should be corrected in the case of a rectangular excitation.

To evaluate the accuracy of the amplitude $\Delta T_{\text {mod }}$, different methods are compared (see Fig. 5). In this case, the sample is excited by a rectangularly modulated field. More details are provided on the four methods in the Supplemental Material [15]. In method 1 the amplitude is determined after a hand analysis of the temperature oscillations measured by the IR camera. We consider that this method leads to an accurate value of the amplitude modulation. In method 2 the signal is demodulated with a Fourier transform of the signal. Method 3 is the same as method 2 but an optimization routine on the modulation frequency that maximizes the Fourier integral is used. In method 4 the temperature signal is fitted with use of the heat-diffusion

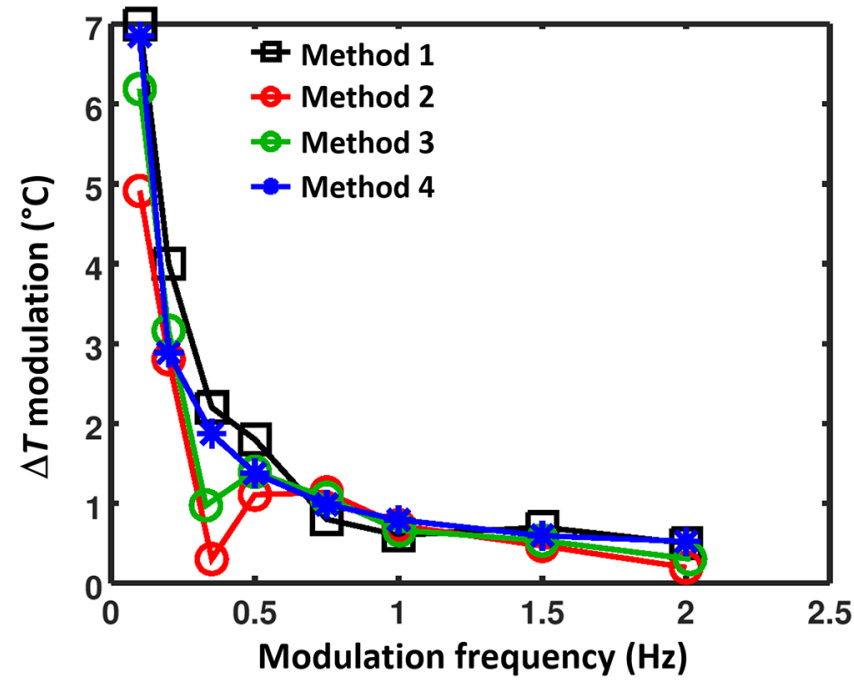

FIG. 5. Extraction of the modulation heating from method 1 (black), method 2 (red), method 3 (green), and method 4 (blue). See the text for an explanation of the four methods. equation [Eq. (13) with a square-wave modulation instead of the $\Lambda(w, \phi)$ triangle function]. In the case of method 4 , the physical constants $\rho, C_{p}$, and $e$, intrinsic to the material are fixed and the adjustable parameters are the absorbed power $P_{\text {abs }}$ and the heat-transfer coefficient $h$. The frequency is fixed to its value deduced from method 3.

The amplitudes $\Delta T_{\text {mod }}$ determined by the four different methods are shown and compared in Fig. 5. It appears that method 2 is not very accurate in the case of large average heating and underestimates the real heating. Frequency optimization gives better results for the reasons explained above and in the Supplemental Material [15]. Finally, the direct fit based on the heat equation gives the best results. We note that method 4 is less sensitive to experimental artefacts than methods 2 and 3, as illustrated by the data obtained at $f_{\text {mod }}=0.35 \mathrm{~Hz}$ (see Fig. 5), which contain several frame drops. Nevertheless, method 4 is the most resource consuming and is used only in the case of pixel processing, not for full-frame demodulation. Full-image demodulation is done with method 3 .

\section{RESULTS AND DISCUSSION}

A transverse-electromagnetic (TEM) transmission-line setup [Fig. 6(a)] was previously used at ONERA to image a microwave magnetic field with the EMIR method [12]. We use this line in preliminary IR thermography experiments to measure the magnetic losses of the samples and test the response of the film in static and modulated experiments. Then two types of thermofluorescence experiments are performed and are presented below.

\section{A. IR thermography}

A complete study of the dependence of the heating power coefficient $\left(\mathrm{K} \mathrm{W}^{-1}\right)$ as a function of the transmission-line input power and the field modulation frequency (square wave) is shown in Figs. 6(b)-6(d). For all frequencies, the film heating varies linearly with the TEMtransmission-line radiated power, as shown by the linear regression of the experimental data. The slope is different depending on the frequency of the rf field and increases with frequency. Figure 6(c) summarizes the evolution of the heating power coefficient $\left(\mathrm{K} \mathrm{W}^{-1}\right)$ as a function of the frequency. The linear behavior is consistent with the dependency of the fraction of energy absorbed by a Tokin film in the frequency range from 0.92 to $3.36 \mathrm{GHz}$ given by the manufacturer (see Fig. SI5 in the Supplemental Material [15]).

To check the validity of the frequency dependence of Eq. (18), we measure the heating amplitude of a Tokin film (E300) for different modulation frequencies of the field [Fig. 6(d)]. The experimental data are in good agreement with theory, with heating inversely proportional to the modulation frequency. Moreover, the average heating of 

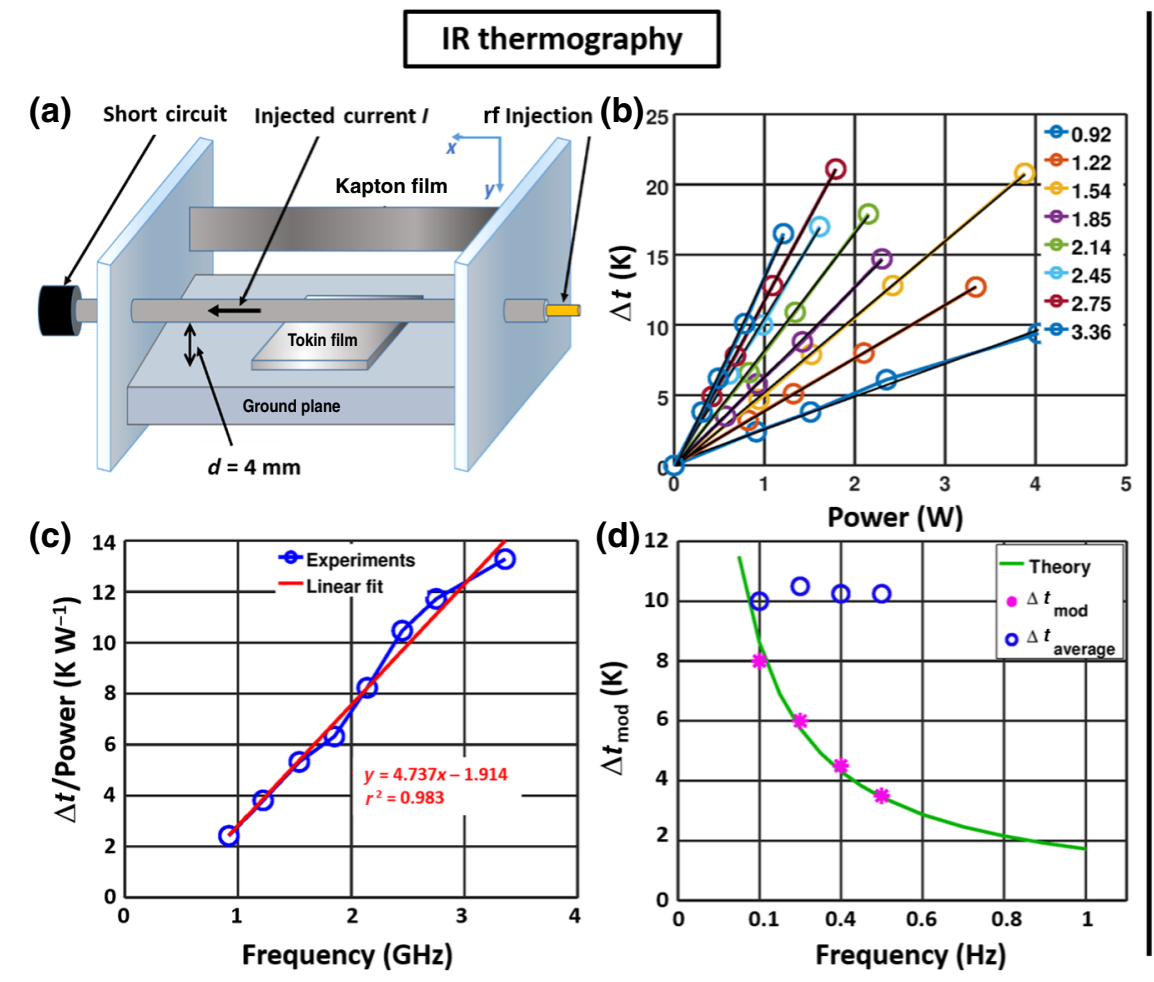

\section{Thermofluorescence}

(e)
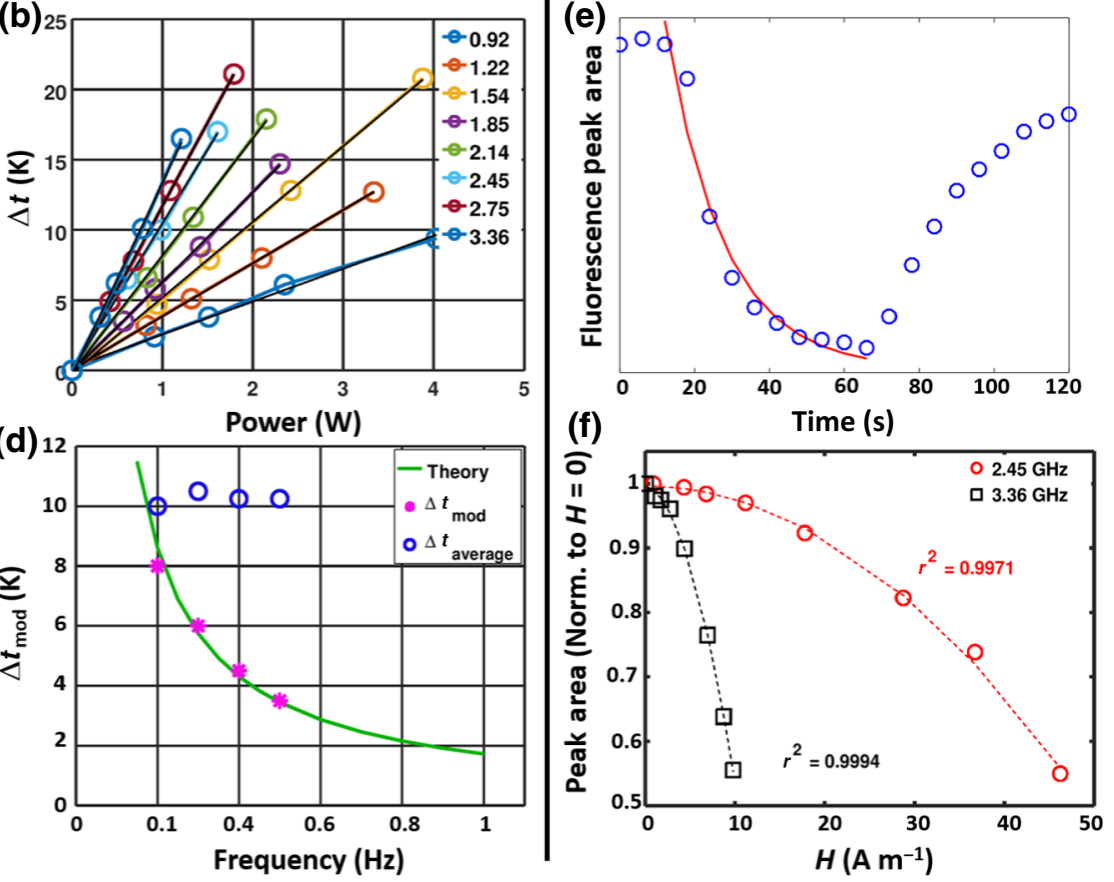

FIG. 6. (a) TEM short-circuited line with both a Kapton film and a Tokin film for simultaneous measurement of $E$ and $H$. (b) Tokinfilm heating for different excitation powers $(1-5 \mathrm{~W})$ and different frequencies $(0.92-3.36 \mathrm{GHz})$. Frequencies are provided in the legend of the graph. (c) Tokin heating power as a function of frequency. The red line is a linear fit of the data. (d) Heating amplitude (magenta stars) and average temperature (blue circles) of a Tokin film (E300) as a function of the modulation frequency of the field. The green curve is the theoretical model given by Eq. (18). (e) Time evolution of the fluorescence peak area of a Tokin film coated with a rhodamine B thin film under a 3.36- $\mathrm{GHz}$ magnetic field $(P=20 \mathrm{~W})$. (f) Fluorescence relative variation at thermal equilibrium as a function of the magnetic field amplitude at $2.45 \mathrm{GHz}$ (red circles) and $3.36 \mathrm{GHz}$ (black squares). The dotted lines are a second-order polynomial fit, with respective correlation factor $R^{2}$ written beside the curves.

the film over time, which corresponds to the case of heating without modulation, is independent of the modulation frequency, as expected.

Equation (18) can also be used to estimate the total thickness of the film in the case where only this parameter is varied. Indeed, the ratio between the heating amplitude of two identical films (with the same conductivity, heat transfer coefficient, etc.) is equal to the ratio of the film thicknesses. Figure SI8 in the Supplemental Material [15] shows the variation of heating under $2.75-\mathrm{GHz}$ excitation as a function of the modulation frequency $(0.1-2 \mathrm{~Hz})$ for four types of Tokin samples of different thicknesses. The latter are a $300-\mu \mathrm{m}$ Tokin film with a rhodamine B polymer film on its surface ( $e=300 \mu \mathrm{m}$ plus polymer thickness). The entry parameters for the data fit use an excitation power of $0 \mathrm{dBm}$ with a correction factor that accounts for the difference between the fraction of absorbed energy at $2.75 \mathrm{GHz}$ (see Fig. SI5 in the Supplemental Material [15]) of the different samples. Other Tokin parameters are given in Table SI2 in the Supplemental Material [15]. The thickness found with the fit (see Fig. SI8 in Supplemental Material [15]), labeled by the letter $e$ in the legend for each curve before the name of the sample, is in very good agreement with the data from the manufacturer. We must consider this result carefully as the fit is very sensitive to the entry parameters and we do not know the value of the excitation power with sufficient accuracy. However, comparison of the fit for the Tokin film without a coating and with a coating yields a thickness of about $5 \%$ of the Tokin-film thickness for the fluorescent coating. This confirms our hypothesis to consider the fluorescent layer as thin from the thermal point of view. Hence, this experiment also validates the capacity of the film to measure magnetic field fluctuations and can be used to image them.

\section{B. Thermofluorescence spectroscopy}

It is important to validate the thermofluorescence method to check that the fluorescence response time is short compared with the temperature time variation. The fluorescence time evolution is measured for a $3.36-\mathrm{GHz}$ magnetic field excitation and various radiated powers (see Fig. SI6 in the Supplemental Material [15]). The fit of the spectra gives the intensity, full width at half maximum, 
and area of the emission peak. The evolution of the peak area as a function of time is shown in Fig. 6(e). Below $P=0.54 \mathrm{~W}$, almost no variation of the fluorescence is detectable (see Fig. SI6 in the Supplemental Material [15]). For other powers, the elevation of temperature induced on the Tokin film is sufficient to trigger a decrease of fluorescence. The fit of the time evolution of the fluorescence peak area with an exponential decay function gives an estimate of the response time of the Tokin film. The different times extracted for different excitation powers at $3.36 \mathrm{GHz}$ are shown in Fig. SI7 in the Supplemental Material [15]. For small heating (low excitation powers), the fluorescence signal is noisy and the uncertainty on the response time is large. The average thermal time constant measured by fluorescence $\left\langle\tau_{\text {fluo }}\right\rangle$ is approximately $14 \mathrm{~s}$ at 2.45 and $3.36 \mathrm{GHz}$ (see Table SI3 in the Supplemental Material [15]). A similar study by IR thermography gives an average thermal time constant $\left\langle\tau_{\mathrm{IR}}\right\rangle$ of approximately $9.6 \mathrm{~s}$ at these two frequencies (see Table SI3 in the Supplemental Material [15]). The discrepancy between the two values is certainly due to the quite large scattering of the time constants deduced from thermofluorescence experiments at low power (see Fig. SI7 in the Supplemental Material [15]). This scattering could be explained by a lack of sensitivity of rhodamine B in thermofluorescence experiments compared with IR thermography especially at low excitation powers that results in a low signal-to-noise ratio and degradation of the quality of the fit. Nevertheless, both methods show a film thermal time constant independent of the field frequency. The film thermal time constant gives also a lower limit to the modulation frequency that can be used (typically $0.1 \mathrm{~Hz}$ ).

In a second type of experiments, we measure the variation of the fluorescence peak area after the thermal equilibrium is reached for different amplitudes of the magnetic field [Fig. 6(f)]. The fluorescence under a magnetic field is normalized to the fluorescence measured without any field. It shows a quadratic dependence of the fluorescence, irrespective of the field frequency. In conclusion, the fluorescence varies linearly with the temperature (calibration curve) and quadratically with the magnetic field power, in agreement with Eq. (4). These experiments show that the magnetically sensitive thermofluorescent films behave as expected and can safely be used for imagery experiments.

\section{Magnetic field imagery by thermofluorescence}

A series of measurements are done on a ZOR antenna [Fig. 7(a)] under square signal modulation to image the magnetic field [10]. We also use these data for sample calibration and fluorescence-to-temperature conversion. The thermal demodulated image obtained with the IR camera [Fig. 7(c)] shows the different magnetic nodes in the near-field region. The maximal heating at $0.2 \mathrm{~Hz}$, located at the central node, is $\Delta T_{\bmod }=9{ }^{\circ} \mathrm{C}$. The main node is surrounded by secondary nodes appearing as spots of lesser intensity on the thermograph. It is possible to visualize structures of the field corresponding to heating below $0.8^{\circ} \mathrm{C}$. Similarly, the demodulation of the fluorescence frames with method 3 results in a thermofluorescent map
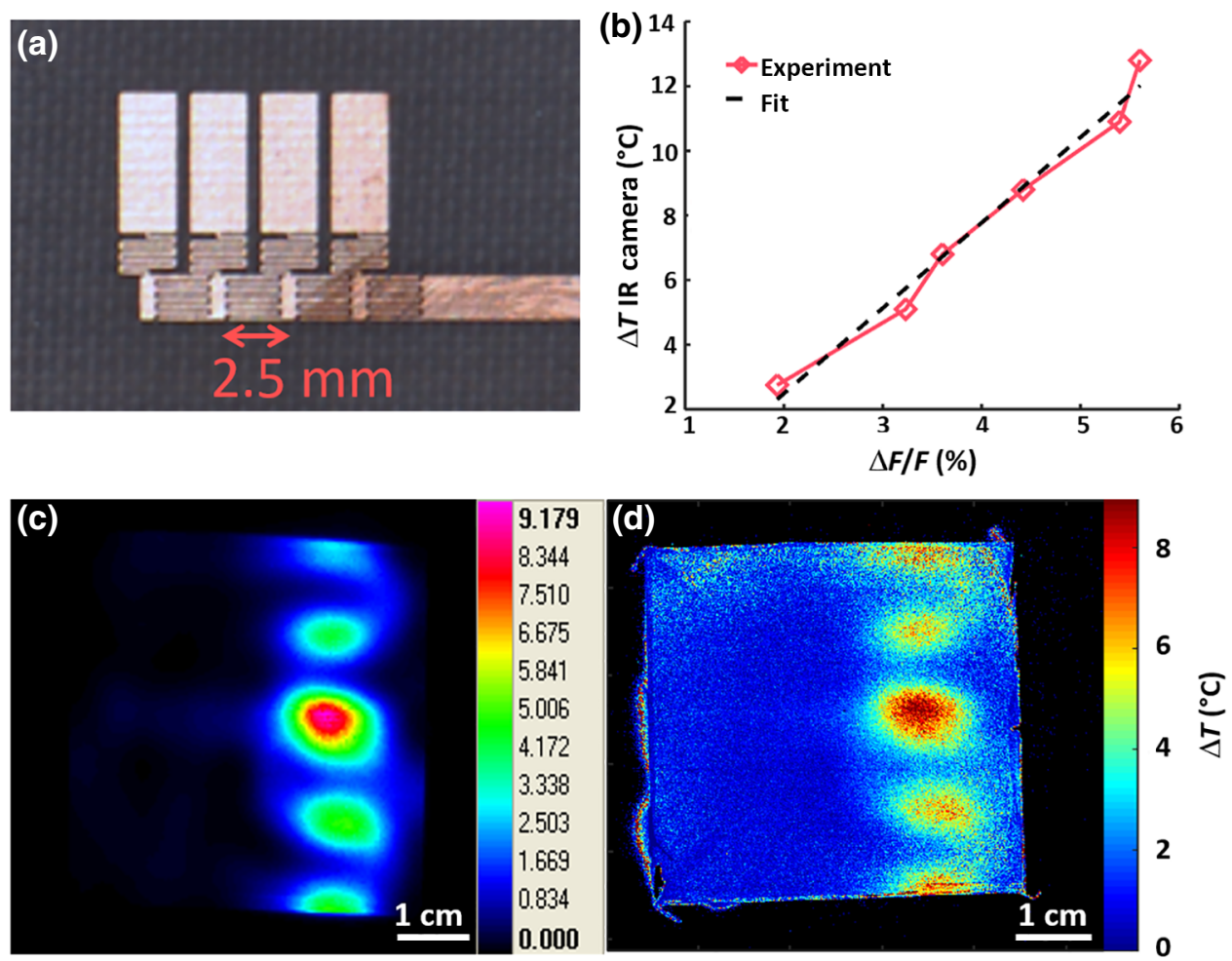

FIG. 7. (a) A view of a ZOR antenna. (b) Calibration curve giving the film temperature heating seen by IR thermography as a function of fluorescence relative variation. The data are from the zone with the greatest heating in the sample. Magnetic near-field imagery by (c) IR thermography and (d) thermofluorescence after signal demodulation. 
of the magnetic field [Fig. 7(d)]. By comparison with the previous image, it is easy to recognize the principal and secondary nodes of the field. Careful observation allows one to see field patterns corresponding to heating less than $2{ }^{\circ} \mathrm{C}$. The resolution is not as good as in the case of the IR thermograph. Plotting the heating of the main mode seen by thermofluorescence as a function of the heating measured by IR thermography for different modulation frequencies gives the calibration curve [Fig. 7(b)]. The curve is fitted by a first-order polynomial equation. This law can then be implemented in the demodulation algorithm to convert the fluorescence relative variation into heat for other experiments using the same film. The visiblethermography image with 470-nm LED array excitation obtained on a seven-digit ZOR antenna shows similar results [18]. A comparison of the image profiles collected by the EMIR and EMVI methods in Ref. [18] leads to a detection threshold estimated as approximately $0.1^{\circ} \mathrm{C}$. The greater sensitivity may be explained by the stronger irradiance of the film. Both results demonstrate the possibilities offered by thin-film technology to image quickly (about $1 \mathrm{~min}$ ) the magnetic field of an antenna.

\section{Electric field measurement of an antenna by in situ probing}

The measurement of fluorescence with a probe, used at the same time as a path for the excitation light and the detection, is very convenient. Indeed, our probe is nonmagnetic, is flexible, and behaves like a local probe. We demonstrate now that putting the fluorescent electrosensitive film at the end of the fiber allows one to realize a thermofluorescent probe. The antenna is an original multiple-feed-per-beam focal-array architecture based on interleaved couplers provided by the Centre National d'Études Spatiales [Fig. 8(a)]. Each three-dimensional directional coupler is composed of one circular waveguide surrounded by six identical circular waveguides. The peripheral waveguides are connected to the central one through thin radial rectangular waveguides, which form coupling slots between waveguides [25]. The complex distribution of the electric field power density $P(x, y)=$ $10 \log \left[\Delta T(x, y) / \Delta T_{\max }\right](\mathrm{dB})$ at the output of the antenna in the near-field region is characterized by the EMIR method and compared with simulations [Fig. 8(d)]. The agreement between both images is very good and proves the efficiency and usefulness of EM-field-imagery methods for characterization. However, it is still very hard to probe the field in such contiguous spaces as the interior of the beams. Moreover, the introduction of any metallic pieces or sensors is prohibited. This is where our method is interesting.

A piece of Kapton film (sensitive to the electric field) coated with a rhodamine B fluorescent thin film is fixed at the entrance of the fiber [Fig. 8(c)]. This thermofluorescent nonmetallic probe is thin enough to be inserted inside the
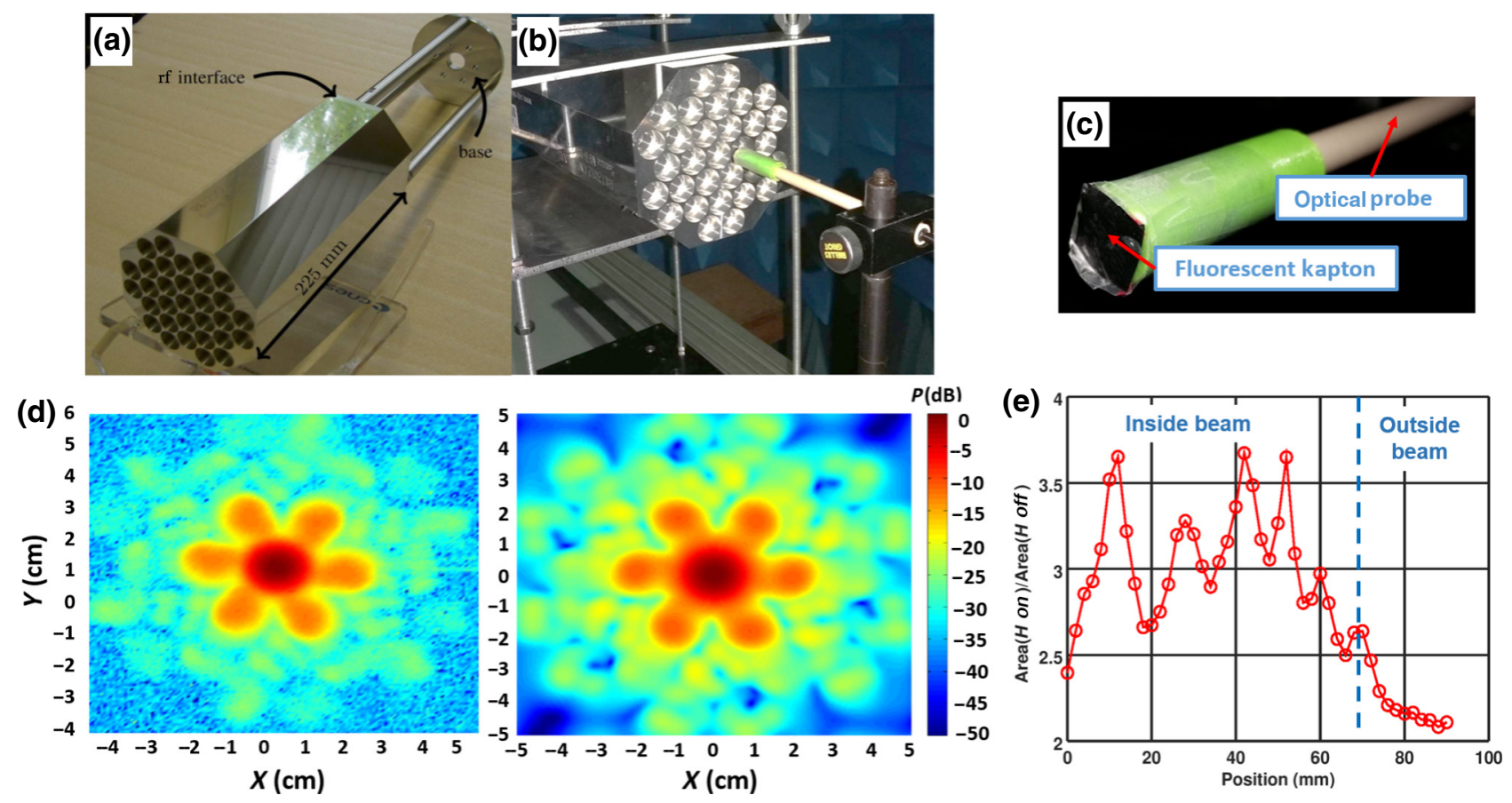

FIG. 8. (a) Multiple-feed-per-beam-focal-antenna mockup [25]. (b) Antenna fixed on a linear motorized stage; the probe is fixed. (c) Thermofluorescent local probe constituted of a Kapton film coated with a rhodamine B fluorescent film, fixed at the edge of the optical fiber entrance. (d) Electric field surface power density in the near-field-region EMIR image (left) and simulation (right). (e) Fluorescence-peak-area variation (normalized to $E=0$ ) as a function of the position of the probe along the central beam axis. 
antenna, is sensitive to local oscillations of the field, and as an all-optical fiber, has the flexibility to carry the luminous signal to the spectrometer. The antenna is mounted on a linear motorized stage so that it can be moved in small steps [Fig. 8(b)]. The probe is motionless. The probe inserted in the central beam measures the electric field $(18 \mathrm{GHz})$ every $2 \mathrm{~mm}$ along the beam axis in the cases where the field is off and on. In the absence of the field, the Kapton is at room temperature. The fluorescence peak area, normalized to the field-off situation, varies quadratically with the electric field amplitude via heating of the Kapton. Figure $8(\mathrm{e})$ shows the fluorescence-peak-area variations along the central beam. The fluctuations of the electric field magnitude are the nodes and antinodes of the field standing wave along the waveguide. Since we measure the magnitude of the field $|E|^{2}$, the nodes are separated by half the wavelength. Inside the antenna [position 0-42 $\mathrm{mm}$ in Fig. 8(e)] the nodes are separated by about $15 \mathrm{~mm}$, half the guide wavelength $\lambda_{g}=30 \mathrm{~mm}$. Close to the output of the waveguide, the wavelength shifts gradually from $\lambda_{g}$ to $\lambda_{0}=c / f=16.6 \mathrm{~mm}$ the free-space wavelength at the operating frequency $f$ (even though the waveguide diameter changes close to the exit), and the node separation is about $8.5 \mathrm{~mm}\left(\lambda_{0} / 2\right)$. Moreover, an exponential decrease of the field amplitude is visible when the probe goes outside the beam. This experiment shows only qualitative results but demonstrates the wide range of possible applications offered by thermofluorescence detection. Indeed, since the sensor is nonmagnetic and nonmetallic, it can be used to probe inside an antenna or waveguide but is also compatible with biological organisms.

\section{E. Discussion}

The development of structured films is the key to largescale EM-field imaging. In particular, the design of thin films with large ferromagnetic losses remains challenging. The Kapton and Tokin films we use allow broadband microwave absorption; since the absorbed power density increases with the square root of the induction frequency, it favors the use of higher induction frequencies. Access to a broader frequency range is a major point. In the doublelayer approach the heat source (absorbent) and heat sensor (fluorophore) are uncoupled, but we can imagine introducing nanoparticles in a polymer that would behave as local EM-field sensors, with fluorophores grafted at their surface, which would then be used to probe the temperature. In such a configuration, it has been shown that the temperature increase could be quite large, the fluorescence varying in spite of the absence of any global heating of the sample [26]. Such a design is now possible and opens the way to the use of spray, foam, or paint, which are much more versatile than films. It could also permit an increase of the signal-to-noise ratio of the detector and access to a very large frequency band.
The thermofluorescent film used in this work is already operational for qualitative imagery applications such as nondestructive testing. We illustrate the potentiality of EMVI by one-dimensional electric field imaging and 2D magnetic field imaging, but these are not limitations and both the electric field and the magnetic field can be probe in one dimension (local probe) or two dimensions (largescale imaging). Concerns remain about quantitative evaluation of the field. In IR thermography uncertainty may remain about the emissivity of the film as it depends on the IR wavelength and on the temperature of the body. A bias exists equally in both IR thermography and the fluorescence method to correlate the temperature variation with the amplitude of the probed field. All experiments assume that all the absorbed energy is converted into heat, and that the heat is directly related to the amplitude of the field. However, to cite an example, in the case of magnetic losses, the energy dissipation of magnetic elements (clusters, nanoparticles, etc.) in oscillating magnetic fields is strongly nonlinear, depending on the field amplitude and frequency $[27,28]$. Such behavior is now well understood and should be integrated in the data postprocessing to properly recover the field amplitude from the temperature.

EMVI-method performances are limited first by fluorophore sensitivity, the magnitude of the thermal dependence varying from dye to dye. Rhodamine $\mathrm{B}$, with an intensity reduction rate of up to $2 \%{ }^{\circ} \mathrm{C}^{-1}$ [29], or organic dyes (intensity reduction rate of $3 \%{ }^{\circ} \mathrm{C}^{-1}$ ) have a strong temperature sensitivity $\left(0.1^{\circ} \mathrm{C}\right)[29,30]$. Other fluorophores, such as quantum dots and rare-earth ions, with even greater sensitivities can also be used [29]. They remain in any case lower than the sensitivity of current IR camera sensors, which can reach $10-\mathrm{mK}$ resolution in the best case. However, IR-sensor spatial resolution is considerably lower than that of optical cameras, mostly $160 \times 120$ pixels or $320 \times 240$ pixels, up to $1024 \times 768$ pixels [31] for the most expensive models working in the medium-wavelength-IR spectral range $(3-5.6 \mu \mathrm{m})$.

A second potential limiting factor of EMVI could be the fluorescence intensity: weak intensity requires a long integration time during camera acquisition and could limit the modulation frequency. Since fluorescence intensity is proportional to the excitation power, one should use a highpower source without dazzling the fluorophore. Intensity is also affected by the fluorophore concentration and the film thickness. In some of our samples, after optimizing the source and the film thickness, we could achieve a $15-\mathrm{ms}$ acquisition time with a framerate of 10 frames/s (camera limitation). For comparison, the framerate usually used with EMIR imaging is $50 \mathrm{~Hz}$. Hence, we can guess that no fundamental limits could prevent EMVI from become as fast as EMIR imaging.

Finally, the long-term stability of the fluorophore could be an issue. Generally, chemical reactions with the environment can alter the fluorescence signal (quenching) as 
can long exposure to UV light (photobleaching) and can degrade the film. However, quenching occurs preferentially in solution, and photobleaching can be avoided by optimization of the exposure time. In our samples, rhodamine B is embedded in a neutral polymer, and is found stable for a long time when not exposed to light excitation that would cause photobleaching [32] or high temperature that would degrade its mechanical or chemical properties.

\section{CONCLUSION}

We demonstrate experimentally the possibility to perform large-scale imaging of the electric or magnetic component of the field as well as local measurements. To do so, the technique of thermofluorescence based on the relative variation of a fluorescence signal as a function of the temperature is used. The temperature is the result of electric or magnetic losses of an absorbent. Thus, this method relies on the coupling of an absorbent sensitive to either the electric field or the magnetic field with a temperaturesensitive fluorescent molecule. This technique, which we call EMVI, shares a lot of points with the EMIR technique, on which it is based. Indeed, both techniques use a nonintrusive film that absorbs a small part of the EM radiation. However, EMVI uses visible-light emission as the transmitter of information.

This method reduces greatly the cost of the detector, and the fluorescence technology would enable large diffusion in fields that were limited to professionals until recently due to the cost of IR technology. Moreover, this work opens the door to the development of magnetic films sensitive to the magnetic field based on ferromagnetic or superparamagnetic nanoparticles, which might display increased sensitivities at a local scale and could also be sprayed on surfaces. The thermofluorescence technique is compared with the EMIR method, with satisfactory results. Then, we show temperature mapping and field mapping with good sensitivity and spatial resolution. The passage from qualitative to quantitative measurements remains challenging and will be part of our future work.

\section{ACKNOWLEDGMENTS}

This work was partly supported by the NEXT Laboratory of Excellence (Université de Toulouse, CNRS). The authors thank the Centre National d'Études Spatiales (French Space Agency) for providing the multiplefeed-per-beam antenna for testing and for the near-field simulation results.

[1] D. Prost, F. Issac, M. Romier, and D. Belot, in presented at the 38th ESA Antenna Workshop (2017).

[2] P. Ripka, Magnetic sensors and magnetometers, Meas. Sci. Technol. 13, 645 (2002).
[3] S. Tumanski, A review of magnetic sensors, Przegl. Elektr. 80, 74 (2004).

[4] H. I. Bassen and G. S. Smith, Electric field probes - A review, IEEE Trans. Antennas Propag. 31, 710 (1983).

[5] J. Lenz and A. S. Edelstein, Magnetic sensors and their applications, IEEE Sens. J. 6, 631 (2006).

[6] C. Ibarra-Castanedo, M. Genest, S. Guibert, J. M. Piau, X. P. V. Maldague, and A. Bendada, in proc. of SPIE - The International Society for Optical Engineering (2007).

[7] S. Huth, O. Breitenstein, A. Huber, and U. Lambert, Localization of gate oxide integrity defects in silicon metaloxide-semiconductor structures with lock-in IR thermography, J. Appl. Phys. 88, 4000 (2000).

[8] G. Breglio, A. Irace, L. Maresca, M. Riccio, G. Romano, and P. Spirito, Infrared thermography applied to power electron devices investigation, Facta Universitatis, Series: Electronics and Energetics 28, 205 (2015).

[9] P. Levesque, J. Grando, F. Issac, M. Nacitas, and D. Balageas, in Journée d'étude SEE sur les capteurs électromagnétiques et leurs applications (Toulouse, 1997).

[10] T. Crépin, F. Issac, S. Bolioli, and D. Prost, Microwave electric field imaging of metamaterials using thermoemissive films, IEEE Antennas Propag. Mag. 56, 37 (2014).

[11] P. Levesque and L. Leylekian, Capteur Vectoriel de Champs Électromagnétiques par Thermographie Infrarouge, French Patent no. 9816079 (1998).

[12] J. Vernières, J. F. Bobo, D. Prost, F. Issac, and F. Boust, Microwave magnetic field imaging using thermo-emissive ferromagnetic micro-structured films, IEEE Trans. Magn. 47, 2184 (2011).

[13] O. Breitenstein, Lock-in IR thermography for functional testing of solar cells and electronic devices, Quant. Infrared Thermogr. J. 1, 151 (2004).

[14] P. E. Nordal and S. O. Kanstad, Photothermal radiometry, Phys. Scr. 20, 659 (1979).

[15] See Supplemental Material at http://link.aps.org/supple mental/10.1103/PhysRevApplied.11.054084 for additional information on experimental setups, film fabrication, demodulation method and film characterization by fluorescence and IR thermography.

[16] J. Lou, T. M. Finegan, P. Mohsen, T. A. Hatton, and P. E. Laibinis, Fluorescence-based thermometry: Principles and applications, Rev. Anal. Chem. 18, 235 (1999).

[17] S. Faure, J. F. Bobo, J. Carrey, F. Issac, and D. Prost, Composant sensible pour dispositif de mesure de champ électromagnétique par thermofluorescence, procédé de mesure et de fabrication correspondants, French Patent no. 1758907 (2017).

[18] H. Ragazzo, S. Faure, J. Carrey, F. Issac, D. Prost, and J. F. Bobo, Detection and imaging of magnetic field in the microwave regime with a combination of magnetic losses material and thermofluorescent molecules, IEEE Trans. Magn. 55, 6500104 (2019).

[19] R. F. Kubin and A. N. Fletcher, Fluorescence quantum yields of some rhodamine dyes, J. Lumin. 27, 455 (1982).

[20] D. Magde, G. E. Rojas, and P. Seybold, Solvent dependence of the fluorescence lifetimes of xanthene dyes, Photochem. Photobiol. 70, 737 (1999).

[21] D. Prost, F. Issac, F. Lemaître, and J. P. Parmentier, in International Symposium on Electromagnetic Compatibility (EMC EUROPE) (2012). 
[22] L. D. Favro and X. Han, in Sensing for Materials Characterization, Processing and Manufacturing, ASNT TONES, edited by G. Birnbaum and B. A. Auld (1998) 1, 399.

[23] D. Prost, F. Issac, and P. Reulet, PIERS Proceedings (Morocco, Marrakesh, 2011).

[24] S. Bagchi and S. K. Mitra, in Nonuniform Sampling: Theory and Practice, edited by F. Marvasti (Kluwer/Plenum, 2001); L. Ying, Sparse Fourier transform via butterfly algorithm, SIAM J. Sci. Comput. 31, 1678 (2009); E. Candès, L. Demanet, and L. Ying, A fast butterfly algorithm for the computation of Fourier integral operators, Multiscale Model. Simul. 7, 1727 (2009); J. Y. Liu, Y. Wang, and J. M. Dai, Research on thermal wave processing of lock-in thermography based on analyzing image sequences for NDT, Infrared Phys. Technol. 53, 348 (2010).

[25] C. Leclerc, M. Romier, H. Aubert, and A. Annabi, K $\alpha-$ band multiple feed per beam focal array using interleaved couplers, IEEE Trans. Microwave Theory Tech. 62, 3 (2014).

[26] H. Huang, S. Delikanli, H. Zeng, D. M. Ferkey, and A. Pralle, Remote control of ion channels and neurons through magnetic-field heating of nanoparticles, Nat. Nanotechnol. 5, $602(2010)$.

[27] D. Soto-Aquino and C. Rinaldi, Nonlinear energy dissipation of magnetic nanoparticles in oscillating magnetic fields, J. Magn. Magn. Mater. 393, 46 (2015); K. Raj and R. Moskowitz, IEEE Trans. Magn. 16, 5 (2003).

[28] J. Carrey, B. Mehdaoui, and M. Respaud, Simple models for dynamic hysteresis loop calculations of magnetic single-domain nanoparticles: Application to magnetic hyperthermia optimization, J. Appl. Phys. 109, 083921 (2011).

[29] D. Jaque and F. Vetrone, Luminescence nanothermometry, Nanoscale 4, 4301 (2012).

[30] M. M. Kima, A. Giry, M. Mastiani, G. O. Rodrigues, A. Reis, and P. Mandin, Microscale thermometry: A review, Microelectron. Eng. 148, 129 (2015).

[31] https://www.flir.fr/browse/professional-tools/thermography -cameras/

[32] D. Ross, M. Gaitan, and L. E. Locascio, Temperature measurement in microfluidic systems using a temperature dependent fluorescent dye, Anal. Chem. 73, 4117 (2001). 